\title{
La agroindustria del agave tequilero Agave tequilana Weber
}

\author{
ANA G. VALENZUELA ZAPATA \\ Departamento de Botánica y Zoología. Universidad de Guadalajara. Franz Schubert 5604, La Estancia, \\ C.P. 45030, Zapopan, Jalisco.
}

\begin{abstract}
Resumen. Las plantaciones del "mezcal azul" Agave tequilana Weber crecen en aproximadamente 43000 hectáreas de tierras sin riego, con 153.6 millones de plantas propiedad de 2500 agricultores en Jalisco. La industria del tequila emplea en la misma entidad a aproximadamente 23202 personas y produce anualmente alrededor de 70 millones de litros que exporta en casi un $90 \%$ a granel a los Estados Unidos y el restante a más de 40 países. El sistema agrícola tradicional ha sido poco estudiado y en los últimos 10 años está cambiando hacia un patrón de monocultivo con uso excesivo de fertilizantes y plaguicidas, cuyas consecuencias no han sido evaluadas. Para algunos agricultores las ventajas son la reducción de 1 a 2 años del ciclo del cultivo; pero la mayoría reconoce el aumento de los costos de producción y los ambientales. Los pequeños productores pierden en la comercialización del agave por una sobreoferta del cultivo y por su incipiente organización social.

Palabras clave: Agroindustria, Agave tequilana, competitividad agrícola, producción, tequila.

Abstract. In Jalisco, the "mezcal azul" Agave tequilana Weber grows in 43000 hectares of non-irrigated farmland representing 153.6 million plants belonging to 2500 owners. The industry of tequila employs 23202 persons in Jalisco and produces 70 million liters per year, exporting $90 \%$ in bulk to the United States and the remainder to more than 40 countries. The traditional cultivation system has barely been studied and has been changing in the last decade, especially to a monoculture with an excessive use of fertilizers and pesticides, but the consequences of their use have not been evaluated. Some producers consider as an advantage the decrease by one to two years in the crop period, but the majority acknowledge productivity and environmental costs. Small growers are losing the agave market due to surplus agave production and their incipient social organization.
\end{abstract}

Key words: Agroindustry, Agave tequilana, agricultural competitivity, production, tequila.

\section{INTRODUCCIÓN}

El sector agroindustrial del agave merece atención por la importancia económica que tiene para Jalisco por la producción y comercialización del tequila y por los ingresos correspondientes en impuestos y exportaciones para el país, sin contar los efectos indirectos que tiene en otras industrias. El objeto de estudio requiere de un análisis dada la situación por la que atraviesa el cultivo del agave, sin una planificación concertada, con la pérdida de germoplasma y una sobreoferta de materia prima, todo esto con consecuencias económicas y sociales. En este diagnóstico se aborda el recurso vegetal y sus sistemas de producción a grandes rasgos, sus regiones de cultivo y su competitividad en un sistema agroindustrial con atractivas expectativas de crecimiento, pero sin acciones deliberadas para fomentar un desarrollo estable.

El enfoque que se utiliza es el de sistemas, donde se define al sistema agroindustrial como un conjunto de estructuras vinculadas entre si por ciertas reglas, no como una secuencia lineal de una entrada y salida de insumos y productos en la cadena de producción y comercialización (Ramos, 1987), donde sus elementos interactúan en un entorno de competitividad. El caso del tequila es, por su específica regionalidad y la falta de usos diversificados de su materia prima, aún mas interdependiente en sus relaciones agroindustriales.

El material para el desarrollo de este análisis son la revisión de estudios al respecto, las experiencias profesionales y de investigación sobre la agroindustria en general y los testimonios personales de agricultores, asociaciones civiles, inversionistas agrícolas e industriales de 1984 a 1995. Para la investigación de los índices agroclimáticos y la zonificación preliminar se revisó la agroclimatología del estado de Jalisco (Villalpando y García, 1993).

\section{LOS CULTIVARES DE AGAVE PARA LA PRODUCCIÓN DE TEQUILA}

El agave tequilero es conocido por sus productores como mezcal, ellos se autonombran mezcaleros y a sus plantaciones las llaman mezcaleras. El término maguey se utiliza sólo para los agaves productores de aguamiel y pulque que no son muy frecuentes en la región. El mezcal azul tequilero, Agave tequilana Weber (Gentry, 1982) es una planta multianual que requiere en promedio de 7 a 10 años como ciclo agrícola. La cosecha total de una plantación se realiza en un periodo de 3 a 4 años después de inhibir la floración ("desquiote"). La madurez óptima para la industria se alcanza de 6 a 12 meses posteriores al corte de la inflorescencia; aunque 
algunas plantas maduran en menor tiempo. La madurez es una característica primordial en la calidad de materia prima que influye en la producción y calidad del tequila (Valenzuela, 1994a). En general los ciclos de cultivo suelen estar afectados directamente por las variables ambientales (región de cultivo); por el manejo agrícola y por la variabilidad de la edad fisiológica y genética de las plantaciones.

Los productores de agave del siglo pasado seleccionaron a la variedad azul (Pérez, 1887), por su menor ciclo de maduración, a sus mejores características industriales y por su mayor producción de vástagos (Valenzuela, 1994b).

A partir de plantas con flor, se han identificado (según Gentry, 1982) en la región de Tequila, Jalisco, 5 de los 9 cultivares reportados el siglo pasado para la producción de tequila; estos corresponden a A.tequilana Weber cv. Azul; A.angustifolia Haw. cv. Sigüín; A. aff.tequilana cv. Moraleño; A. aff.cantala Roxb.cv. Bermejo; A.rhodacantha Trel. cv. Chato o Sahuayo. Los tres primeros son similares en talla y peso del índice de cosecha (cabeza o piña de 25 a $70 \mathrm{Kg}$ ); los dos últimos de grandes dimensiones (cabeza > $70 \mathrm{Kg}$ ) son tardíos para madurar (>9 años). Recientemente se han encontrado plantas jóvenes de un escaso cultivar parecido al Azul pero con franjas amarillas de $1 \mathrm{~cm}$ en los márgenes de las hojas ("Azul listado": A. aff. tequilana, no descrito en la literatura) y de un ejemplar denominado "Criollo" aparentemente una forma de A. angustifolia. Se buscan aun las variedades conocidas como: "Mezcal chino", "Mano larga", "Zopilote" y "Pie o pata de mula" asi como si sus nombres científicos coinciden con los cultivares ya encontrados.

La presión de selección de la variedad azul después de reglamentar su uso en la Norma Oficial Mexicana del tequila (SEC.OFI, 1972), ha disminuido las cantidades de los otros cultivares, a tal grado que en la región de mayor diversidad (Tequila) su frecuencia máxima es aproximadamente del $1 \%$ para las variedades más abundantes.

Los cultivares tradicionales deben ser conservados por representar un banco de genes adaptado a condiciones específicas y susceptibles a ser insertados en los cultivos modernos, además de ofrecer una alternativa de producción de subsistencia donde las razas modernas no son adecuadas (Frankel y Soulé, 1981, en Amo y Caamal, 1992). Agave tequilana en la región de Tequila es tan productivo como una planta $\mathrm{C}_{3}\left(>2 \mathrm{Kg} \mathrm{m}^{-2}\right.$ año $^{-1}$; Nobel y Valenzuela, 1987) y puede desarrollarse en zonas semiáridas (Palomino, 1994) gracias a su Metabolismo Acido Crasuláceo (CAM) pero su productividad y calidad para la industria tequilera bajo estas condiciones no son las deseadas.

\section{CONDICIONES AMBIENTALES DE LAS ZONAS CULTIVADAS}

Los suelos de estas regiones son cambisoles y luvisoles de acuerdo a la clasificación de la FAO (Valenzuela, 1992), de textura franco arcillosa, drenaje eficiente, $\mathrm{pH}$ tendiente a la acidez, generalmente bajos en materia orgánica y de color café a rojizo. La plantación desmedida de los últimos 10 años (18 000 has: Valenzuela, 1985; 50000 has CRT, 1995) ocasionó el crecimiento de zonas cultivadas de agave hacia valles fértiles y pendientes onduladas desmontadas para esta actividad; sin faltar desde luego en fuertes pendientes y en suelos pedregosos.

El clima predominante en la región tequilera de

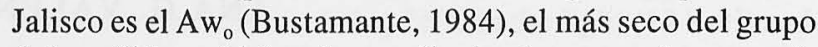
de los cálidos subhúmedos con lluvias de verano. Las aproximadamente 150 millones de plantas en producción (CRT, 1995) se ubican en un rango altitudinal de 800 a $2000 \mathrm{msnm}$ (Cuadro 1), con un promedio de 600 a $800 \mathrm{~mm}$ de precipitación pluvial de junio a octubre y anualmente de 600 a 1200 $\mathrm{mm}$, ambos con una probabilidad de ocurrencia del $70 \%$. Las regiones con una precipitación menor de $500 \mathrm{~mm}$ en la época húmeda han mostrado cosechas de baja calidad y los mayores de $1000 \mathrm{~mm}$ requieren de suelos bien drenados.

Las temperaturas cardinales para el agave tequilero son una mínima de 3 a $10^{\circ} \mathrm{C}$, óptimas diurna/nocturna de $30 /$ $15^{\circ} \mathrm{C}$ (Nobel y Valenzuela, 1987) y máxima entre 30 a $34^{\circ} \mathrm{C}$. Una zonificación altitudinal preliminar constaría de 4 áreas, las de barranca (800-1000 m), las partes bajas (1000-1300 m), las intermedias (1300-1700 m) y las mesetas altas de (1700-2100 $\mathrm{m}$; Fig. 1). Se distinguen como áreas contrastantes las mesetas de la región de Los Altos (2000 m) y los cultivos de la Barranca de Tequila ( $800 \mathrm{~m}$; depresión del río Lerma Santiago) que presentan de 400 a 2000 unidadescalor, en basea $15^{\circ} \mathrm{C}$, índices agroclimáticos que afectan la floración y el proceso de madurez de los cultivos (Villalpando y García, 1993).

\section{CARACTERISTICAS DE LA MATERIA PRIMA DE AGAVE TEQUILANA}

El ciclo del cultivo se acorta en los lugares bajos pero la calidad $^{1}$ de la materia prima deseada por la industria no es la óptima; mientras que en las regiones altas el ciclo se alarga y los parámetros de calidad, como son la cantidad de azúcares reductores y un bajo porcentaje de fibra mejoran entre otras características. Respecto al desarrollo, se observa que las dimensiones de la planta y la zona de crecimiento de hojas o "cogollo" se incrementan con la altitud de la región (Fig. 2). Las Figuras 3 y 4 indican la tendencia de las características de diámetro y altura de "cabeza" y peso de la misma, en tres regiones con altitud ascendente. El peso específico de la materia prima es mayor en las mesetas de Los Altos con un promedio regional de $60 \mathrm{Kg} / \mathrm{c}$ (Peso/ cabeza). En la región intermedia (Atotonilco) se reconocen volúmenes mayores y con un peso promedio de $50 \mathrm{Kg} / \mathrm{c}$ y en la región de Tequila con $40 \mathrm{Kg} / \mathrm{c}$. Los datos coinciden con la experiencia aunque no es una regla general para nichos especiales de la región de Tequila con buenos rendimientos.

\footnotetext{
${ }^{1}$ Los parámetros de calidad del tequila son químicos, físicos y organolépticos, debido a esto una de las industrias de mayor renombre establece que la materia prima para alcanzar su calidad es únicamente de la región de Tequila.
} 
CUADro 1. Principales índices agroclimáticos de las regiones cultivadas con agave tequilero en Jalisco, los índices de humedad están calculados con un 70\% de probabilidad; las localidades y datos se consultaron de Villapando y García, 1993.

\begin{tabular}{|c|c|c|c|c|c|c|c|c|}
\hline \multirow[t]{2}{*}{ Indices agroclimáticos } & \multicolumn{3}{|c|}{ Región de Los Altos } & \multirow[b]{2}{*}{ Zapotlanejo } & \multirow[b]{2}{*}{ Tototlán } & \multicolumn{3}{|c|}{ Región de Tequila } \\
\hline & Arandas & Acatic & Atotonilco & & & $\begin{array}{l}\text { Antonio } \\
\text { Escobedo }\end{array}$ & $\begin{array}{l}\text { Hostoti- } \\
\text { paquillo }\end{array}$ & Sta. Rosa \\
\hline \multicolumn{9}{|l|}{ Datos estación } \\
\hline Altitud (m) & 2020 & 1681 & 1600 & 1596 & 1540 & 1360 & 1291 & 800 \\
\hline Latitud & $20^{\circ} 43^{\prime}$ & $20^{\circ} 47^{\prime}$ & $20^{\circ} 33^{\prime}$ & $20^{\circ} 37^{\prime}$ & $20^{\circ} 32^{\prime}$ & $20^{\circ} 48^{\prime}$ & $20^{\circ} 48^{\prime}$ & $20^{\circ} 55^{\prime}$ \\
\hline Longitud & $102^{\circ} 26^{\prime}$ & $102^{\circ} 54^{\prime}$ & $102^{\circ} 31^{\prime}$ & $103^{\circ} 40^{\prime}$ & $102^{\circ} 48^{\circ}$ & $104^{\circ} 08^{\prime}$ & $104^{\circ} 08^{\prime}$ & $103^{\circ} 43^{\prime}$ \\
\hline \multicolumn{9}{|l|}{ Índices de temperatura } \\
\hline \multicolumn{9}{|l|}{ Junio-octubre } \\
\hline Temperatura media ${ }^{\circ} \mathrm{C}$ & $18-22$ & $18-22$ & $22-26$ & $18-22$ & $22-26$ & $22-26$ & $22-26$ & $>26$ \\
\hline Temperatura máxima ${ }^{\circ} \mathrm{C}$ & $26-30$ & $26-30$ & $26-30$ & $26-30$ & $26-30$ & $26-30$ & $26-30$ & $30-34$ \\
\hline Temperatura mínima ${ }^{\circ} \mathrm{C}$ & $<14$ & $14-18$ & $14-18$ & $14-18$ & $14-18$ & $14-18$ & $14-18$ & $18-22$ \\
\hline Temperatura diurna ${ }^{\circ} \mathrm{C}$ & $22-26$ & $22-26$ & $22-26$ & $22-26$ & $22-26$ & $22-26$ & $26-30$ & $>30$ \\
\hline Temperatura nocturna ${ }^{\circ} \mathrm{C}$ & $14-18$ & $14-18$ & $18-22$ & $18-22$ & $18-22$ & $18-22$ & $18-22$ & $>22$ \\
\hline Unidades calor base $15^{\circ} \mathrm{C}$ & $400-800$ & $800-1200$ & $800-1200$ & $800-1200$ & $800-1200$ & $800-1200$ & $1200-1600$ & $1600-2000$ \\
\hline Unidades fototérmicas base $10^{\circ} \mathrm{C}$ & $1600-2000$ & $2000-4 C 00$ & $2000-4000$ & $2000-4000$ & $2000-4000$ & $2400-2800$ & $2400-2800$ & $2800-3200$ \\
\hline Temperatura máxima ${ }^{\circ} \mathrm{C}$ anual & $22-26$ & $26-30$ & $26-30$ & $26-30$ & $26-30$ & $26-30$ & $>30$ & $>30$ \\
\hline Temperatura mínima ${ }^{\circ} \mathrm{C}$ anual & $<10$ & $10-12$ & $12-14$ & $10-12$ & $10-12$ & $12-14$ & $14-16$ & $16-18$ \\
\hline Horas frio, nov. a feb. & $>600$ & $200-400$ & $<200$ & $<200$ & $<200$ & $<200$ & 0 & 0 \\
\hline
\end{tabular}

Índices de humedad

Junio-øctubre

Precipitación pluvial mm $70 \%$ prob.

Evaporación (mm)

$600-800$

600-800

600-800

600-800

$600-800$

$800-1200$

500-600

600-800

$600-700$

$700-800$

$700-800$

700-800

700-800

$700-800$

700-800

800-900

500-600

600-700

600-700

600-700

500-600

600-700

600-700

$>700$

Índice de humedad 70\% prob.

Días nublados

Adecuado

Adecuado

Adecuado

Adecuado

Adecuado

Adecuado

Mod. efic.

Adecuado

60-75

45-60

60-75

$<30$

60-75

45-60

$<30$

60-75

15-30

$30-45$

45-60

30-45

30-45

45-60

30-45

120-150

120-150

120-150

$>150$

120-150

120-150

90-120

120-150

90-120 60-90

60-90

$60-90$

$30-60$

90-120

30-60

$30-60$

$800-1200 \quad 600-800 \quad 600-800 \quad 600-800$

$600-800$

800-1200

$500-600$

$800-1200$

Periodo libre heladas $80 \%$ prob.

210-240

$240-270>300$

270-300

$>300$

210-240

$>300$

\section{EL SISTEMA AGRÍCOLA DEL AGAVE TEQUILERO}

Existen evidencias de que la cosecha del "mezcal" para el "vino mezcal de Tequila" se realizaba desde 1621, lo cual indica una antigüedad de 350 años de cultivo en la región central del estado de Jalisco (Muriá, 1990). Los sistemas de producción predominantes pueden tipificarse por su ubicación geográfica y densidad de plantación en dos: el más antiguo en la región de Tequila (siglo XVII) con bajas densidades y el de la región de Los Altos (siglo XIX; Luna,
1991) con variantes de 3000 hasta 6000 plantas por hectárea (Valenzuela, 1994a). El manejo agrícola presenta tres grandes etapas: la primera que consiste en preparar y desarrollar la plantación; la segunda en el mantenimiento anual hasta el inicio de la madurez y la tercera que comprende el mantenimiento de la plantación y su cosecha. La relación entre la antigüedad del cultivo y la especialización de labores agrícolas es directamente proporcional, a un mayor conocimiento tradicional, la diferenciación de prácticas es mayor, lo cual se puede observar en los Cuadros 2 y 3 en dos prototipos de manejo agrícola. 

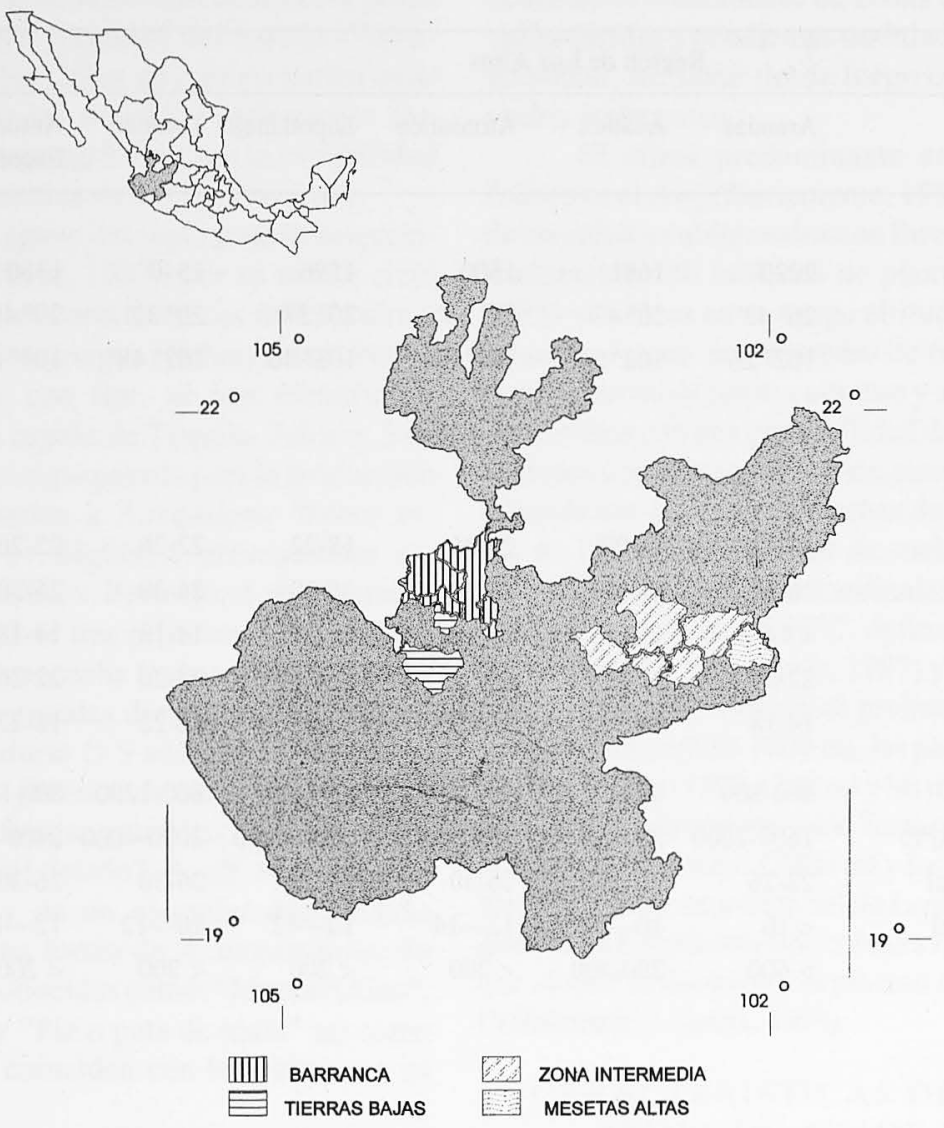

FIGURA 1. Zonas cultivadas con agave tequilero en Jalisco y su delimitación preliminar a partir de índices agroclimáticos.

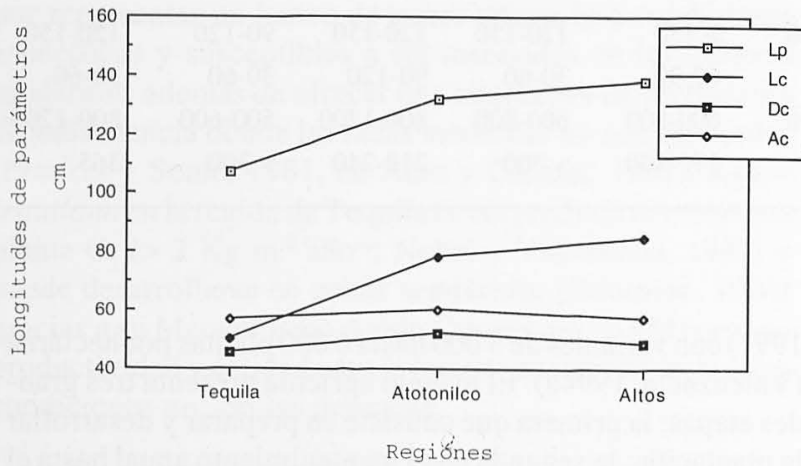

Figura 2. Características de la materia prima de Agave tequilana longitud de planta (Lp); longitud de "cogollo" o zona de crecimiento de hojas (Lc) y diámetro y altura de "cabeza" (Dc, Ac; índice de cosecha) en las regiones de Tequila ( $1280 \mathrm{~m})$, Atotonilco (1600 m) y Altos (2020 $\mathrm{m})$. Los datos se tomaron en plantaciones en cosecha ( $>8$ años de cultivo) con síntomas de madurez en 1991.

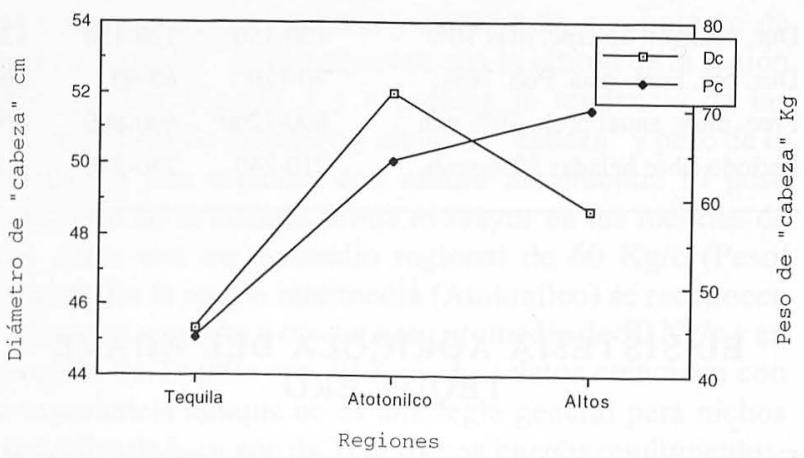

Figura 3. Diámetro y peso de "cabeza" (Dc, Pc; índice de cosecha) de materia prima Agave tequilana en las regiones de: Tequila $(1280 \mathrm{~m})$, Atotonilco $(1600 \mathrm{~m})$ y Altos $(2020 \mathrm{~m})$. Los datos se tomaron en plantaciones en cosecha ( $>8$ años de cultivo) con síntomas de madurez en 1991 . 
CUADRo 2. Labores agrícolas mecanizadas y manuales de la región de Tequila y su forma de realización: convencional (c) o propia (p).

\begin{tabular}{|c|c|c|c|c|c|}
\hline Año de Plantación & Mecanizadas & Manuales & Año de Plantación & Mecanizadas & Manuales \\
\hline Subsuelo y barbecho cruzado & c & & 5o. año de cultivo & & \\
\hline Rastreo & $\mathrm{c}$ & & Aplicación herbicida & & c \\
\hline Acarreo de plantas & c & & Aplicación fertilizantes & & c \\
\hline Plantación & & $\mathrm{p}$ & Aplicación insecticidas y fungicidas & & c \\
\hline Limpia manual & & $\mathrm{p}$ & Arada & c & \\
\hline Aplicación insecticidas y fungicidas & & c & Guardarraya (herbicida) & & c \\
\hline $\begin{array}{l}\text { Guardaraya eliminación de maleza } \\
\text { protección de incendios en límites. }\end{array}$ & & c & Arranque o desahije y prep. hijuelos & & $\mathrm{p}$ \\
\hline 1er. año de cultivo & & & 6o. año de cultivo-cosecha & & \\
\hline Aplicación fertilizantes & & c & Aplicación herbicida & & c \\
\hline Aplicación insecticidas y fungicidas & & $\mathrm{c}$ & Aplicación fertilizantes & & c \\
\hline Arada & $\mathrm{c}$ & & Aplicación insecticidas y fungicidas & & c \\
\hline Guardarraya (herbicidas) & & c & Arada & c & \\
\hline Limpia manual & & $\mathrm{p}$ & $\begin{array}{l}\text { Guardarraya (herbicida) } \\
\text { Poda o barbeo }\end{array}$ & & $\begin{array}{l}\mathrm{c} \\
\mathrm{p}\end{array}$ \\
\hline 2o. año de cultivo & & & Desquiote & & $\mathrm{p}$ \\
\hline Aplicación herbicida & & c & & & \\
\hline Aplicación fertilizantes & & c & 7o. año de cultivo-cosecha & & \\
\hline Aplicación insecticidas y fungicidas & & $\mathrm{c}$ & Aplicación herbicida & & c \\
\hline Arada & $\mathrm{c}$ & & Aplicación fertilizantes & & c \\
\hline Guardarraya (herbicida) & & c & Aplicación insecticidas y fungicidas & & c \\
\hline 3er. año de cultivo & & & $\begin{array}{l}\text { Arada } \\
\text { Guardarraya (herbicida) }\end{array}$ & $\mathrm{c}$ & c \\
\hline Aplicación herbicida & & c & Desquiote & & $\mathrm{p}$ \\
\hline Aplicación fertilizantes & & c & & & \\
\hline Aplicación insecticidas y fungicidas & & c & 80. año de cultivo-cosecha & & \\
\hline Arada & c & & Aplicación herbicida & & c \\
\hline Guardarraya (herbicida) & & c & Aplicación fertilizantes & & c \\
\hline Arranque o desahije y prep. hijuelos & & $\mathrm{p}$ & Aplicación insecticidas y fungicidas & & c \\
\hline 40. año de cultivo & & & Arada & c & \\
\hline Aplicación herbicida & & c & Guardarraya (herbicida) & & c \\
\hline Aplicación fertilizantes & & c & Desquiote & & $\mathrm{p}$ \\
\hline Aplicación insecticidas y fungicidas & & c & Poda o barbeo & & $\mathrm{p}$ \\
\hline $\begin{array}{l}\text { Arada } \\
\text { Guardarraya (herbicida) }\end{array}$ & c & & 9o. año de cultivo-cosecha & & \\
\hline $\begin{array}{l}\text { Guardarraya (herbicida) } \\
\text { Arranque o desahije y prep. hijuelos }\end{array}$ & & $\mathrm{p}$ & Aplicación fertilizantes & & c \\
\hline \multirow[t]{2}{*}{ Poda o barbeo } & & $\mathrm{p}$ & Aplicación insecticidas y fungicidas & & c \\
\hline & & & Cosecha & & $\mathrm{p}$ \\
\hline
\end{tabular}

De las labores que caracterizan a un sistema agrícola tradicional (Hernández, 1993), la mayoría se realizan en el agave tequilero, con excepción a las referidas como ceremonias de agradecimiento por la cosecha. El sistema agrícola tradicional con detallada percepción cultural y manejo de los recursos vegetales (Bye, 1993) se conserva en el agave tequilero pero no se ha investigado en su totalidad ni evoluciona intencionalmente hacia un desarrollo integral.

Los procedimientos y herramientas de labores tradicionales han sido mayormente depurados en la región de Tequila, prueba de ello son los diseños de herramientas de poda (machete "barbeador") con los que se realizan prácticas diferenciadas para diversos objetivos (Valenzuela, 1987; Cuadro 4).
Las alteraciones que han tenido los sistemas de producción de agave conllevan a la mayor mecanización de labores; al uso creciente de fertilizantes y al control químico de malezas, plagas y enfermedades; los productos más aplicados son fertilizantes sólidos, herbicidas, insecticidas y fungicidas (Cuadro 5). Una gran parte de estos productos no están validados por trabajos de investigación, lo cual no es un requisito para los vendedores de agroquímicos, quienes llenan el vacío de la asesoría técnica y recomiendan a los productores. Asimismo los agricultores señalan que la selección de vástagos de buena calidad, el control eficaz de la maleza y la fertilización han acortado entre 1 y 2 años el ciclo de producción; aunque reconocen consecuencias perjudicia- 
CuAdro 3. Labores agrícolas mecanizadas y manuales de la región de Los Altos y su forma de realización: convencional (c) o propia (p).

Año de Plantación Mecanizada Manual

Subsuelo y barbecho
Aplicación abonos orgánicos
Rastreo y surcado
Acarreo de plantas

Plantación

Aplicación de fertilizantes, insecticidas y fungicidas

Aplicación herbicidas

$\begin{array}{ll}\mathrm{c} & \\ \mathrm{c} & \\ \mathrm{c} & \\ \mathrm{c} & \mathrm{p} \\ & \mathrm{c} \\ & \mathrm{c} \\ & \mathrm{c}\end{array}$

Edad de cultivo 1 año Aplicación de herbicida Aplicación fertilizantes Aplicación fungicidas e insecticidas

Del 20. al 3o. año de cultivo

Aplicación de herbicida Aplicación fertilizantes Aplicación fungicidas e insecticidas

Del 4o. al 5o. año de cultivo Aplicación de herbicida Aplicación fertilizantes

Aplicación fungicidas e insecticidas

Poda de acceso (chaponeo)

Desahijes y prep. de hijuelos

c

c

c

Del 6o. al 7o. año de cultivo

Aplicación de herbicida

Aplicación fertilizantes

Aplicación fungicidas e insecticidas

Poda de acceso (chaponeo)

Del 8o. al 110. año de cultivo

Protección de incendios

eliminación de maleza (herbicida)

Cosecha (jima)

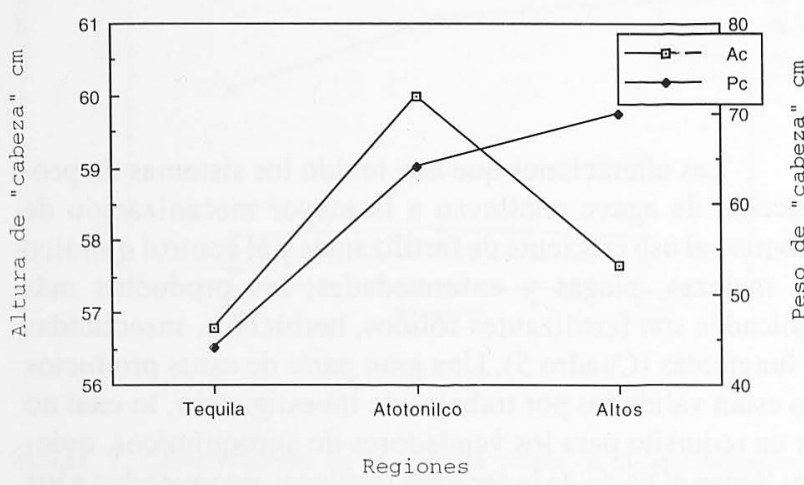

Figura 4. Altura y peso de "cabeza" (Ac, Pc; índice de cosecha) de materia prima de Agave tequilana en las regiones de: Tequila $(1280 \mathrm{~m})$, Atotonilco $(1600 \mathrm{~m})$ y Altos $(2020 \mathrm{~m})$. Datos tomados en plantaciones en cosecha (> 8 años de cultivo) con síntomas de madurez en 1991.
Cuadro 4. Labores especializadas en poda o «barbeo» en Agave tequilana, su intensidad y edad de cultivo en que se realizan (Valenzuela, 1987).

\begin{tabular}{lll}
\hline Labor & Corte & Edad de cultivo \\
\hline $\begin{array}{l}\text { Podas de acceso } \\
\text { a labores } \\
\text { agrícolas. }\end{array}$ & $\begin{array}{l}\text { Hojas laterales } \\
\text { ligero }\end{array}$ & $>3$ años \\
«Cacheteo», & \\
«Chaponeo» & \\
$\begin{array}{l}\text { Podas para «desahije» } \\
\text { arranque de vástagos }\end{array}$ & $\begin{array}{l}\text { Hojas laterales } \\
\text { regular }\end{array}$ & $3-5$ años
\end{tabular}

Preparación de

vástagos.

«barbeo de semilla»

Acentuado en

Para plantación

Podas fitosanitarias

«Barbeo de desemplague»

Ligero a regular $>2$ años

Podas de formación

«Arbolito»

Ligero alrededor

de la planta

$>4$ años

«Farol»

Podas para acelerar madurez

«Barbeo castigado»

Muy acentuado

$>6$ años

les como la mayor incidencia de plagas y enfermedades y el aumento en los costos de producción.

El desconcierto sobre el diagnóstico actual del sistema agrícola se incrementa cuando ante esta problemática no existe una institución de investigación para el apoyo a la actividad agavera; por lo tanto no se conocen medidas deliberadas para plantear su desarrollo tecnológico.

\section{COMPETITIVIDAD DEL SECTOR PRODUCTOR DE AGAVE}

Al analizar las fuerzas competitivas (Thompson y Strickland, 1994) del sector "mezcalero", se entiende como los productos sustitutos de azúcares de caña se han consolidado como una amenaza competitiva para los productores de agave. El sector fragmentado con una gran cantidad de agricultores, el crecimiento lento de la demanda, la ausencia de un mercado opcional para la materia prima y la dificultad para salir del negocio entre otros elementos de competitividad interna; los coloca ante la industria con un débil poder negociador y los conduce a la reducción de precios de venta de agave hasta en N\$150.00 Ton (Fig. 5).

La calidad de la materia prima de la región de Los Altos sobresale por factores ambientales principalmente y los productores obtienen mayores ingresos económicos, sobre todo cuando el precio está basado en la cantidad de azúcares reductores (Cuadro 6) y por las densidades de plantación. La ventaja competitiva de esta región tiende a ser 
CuADro 5. Aplicaciones de agroquímicos y pesticidas en el cultivo de Agave tequilana.

\begin{tabular}{|c|c|c|c|}
\hline Insumo & Aplicación & Forma & Periodo \\
\hline Fertilizantes & $\begin{array}{l}\text { Sólida al suelo } \\
\text { Productos } \\
\text { nitrogenados y } \\
\text { fórmulas NPK }\end{array}$ & Manual & Julio-Agosto \\
\hline Herbicidas & $\begin{array}{l}\text { Preemergentes } \\
\text { Postemergentes }\end{array}$ & $\begin{array}{l}\text { Aspersora manual } \\
\text { Aspersora manual }\end{array}$ & $\begin{array}{l}\text { Junio } \\
\text { Agosto-Septiembre }\end{array}$ \\
\hline Insecticidas & $\begin{array}{l}\text { Plagas del suelo } \\
\text { Plagas en el } \\
\text { cogollo }\end{array}$ & $\begin{array}{l}\text { Aspersora manual } \\
\text { Enterrado en la } \\
\text { cepa } \\
\text { Aspersora manual }\end{array}$ & $\begin{array}{l}\text { Junio-Octubre } \\
\text { Junio-Octubre }\end{array}$ \\
\hline Fungicidas & $\begin{array}{l}\text { Daños en el tallo } \\
\text { y en el cogollo } \\
\text { Phytophora sp. } \\
\text { Phytium sp. } \\
\text { Fusarium sp. }\end{array}$ & Aspersora manual & Junio-Enero \\
\hline
\end{tabular}

NPK: Nitrógeno, Fósforo y Potasio en forma de 17-17-17.

Cogollo: hojas de la parte central que protegen la zona de crecimiento.

duradera, y por lo tanto de riesgo para los agaveros de la región de Tequila, quienes han observado la migración del cultivo y el ingreso constante de la materia prima de Los Altos a la planta industrial de Tequila. Solamente 2 fábricas de la región de Tequila prefieren el agave ahí producido; las 13 fábricas de Los Altos no consumirían materia prima de otra región y las restantes 20 prefieren el de la región de Los Altos (Fig. 6).

El fenómeno cíclico de la sobreoferta y déficit de agave tequilero (1900:60 000 has., 1940:2 000; Luna, 1991) parece repetirse indefinidamente, asi como la falta de su planeación y estrategias alternativas de desarrollo. Los más de 2000 productores de agave pertenecen a 5 organizaciones que por su historial se consideran inoperantes (Luna, 1991) y que a partir de 1995 se mantienen en fricciones continuas (Siglo 21, 1995). Aunado a esto, el sector agroindustrial se ha mantenido poco regulado, la planta industrial se convierte en maquiladora de tequila para los Estados Unidos (Luna, 1991) y la Norma Oficial Mexicana (SECOFI, 1993) es ambigua para verificar la autenticidad de la bebida. En el aspecto agrícola se carece de información de este cultivo en los censos agrícolas oficiales (Valenzuela, 1994a), de investigación, de diversificación de usos e innovación de productos y del conocimiento de la demanda real anual del complejo industrial.

\section{EL SISTEMA INDUSTRIAL DEL TEQUILA}

La actividad económica de A. tequilana es una unidad agroindustrial protegida por una denominación de origen como el cogñac y el champagne desde 1974 en el acuerdo de Lisboa (Pla y Tapia, 1993). En 1994 se exportó a granel casi

\section{PRODUCTOS SUSTITUTOS}

Azúcares de caña y sorgo

Son una amenaza para el productor de agave por su precio Los costos del cambio a un mayor consumo de agave son fuertes, pues para los otros azúcares las estructuras son de dilución o mezcla; para el agave son hornos, calderas, molinos y exprimidores.

\section{RIVALIDAD ENTRE VENDEDORES DE MATERIA} PRIMA EN COMPETENCIA POR:

\section{Aumento de productos de agave tequilero}

Crecimiento muy lento de la demanda

Crecimiento muy lento de la demanda de plantaciones

Tendencia a la reducción de precios

No hay dificultad de la industria por cambiar de productor Es difícil salirse del negocio

No hay demanda de la materia prima en el extranjero

Figura 5. Principales fuerzas en el entorno competitivo de la venta de materia prima de agave tequilero.

43 millones de litros, (94\%) un 90\% de estos a los Estados Unidos y un $6 \%$ envasado (CRIT, 1994; Fig. 7). En el proceso industrial con la cocción se hidroliza la inulina de la materia prima de agave y se extraen las mieles para la fermentación de los azúcares solos (tequila 100\% de agave) o mezclados con azúcares de caña (tequila mixto 51-49\%); posteriormente se destilan y se envasan sin madurarse (te- 
Cuadro 6. Precio de la tonelada de materia prima de agave tequilero. (CRT, Comunicación personal, 1995).

\begin{tabular}{lc}
\hline \% de Azúcares reductores & $\begin{array}{c}\text { Precio por Tonelada } \\
\text { N\$ }\end{array}$ \\
\hline $16.0-18.9$ & 390.00 \\
$19.0-19.9$ & 450.00 \\
$20.0-20.9$ & 470.00 \\
$21.0-21.9$ & 500.00 \\
$22.0-22.9$ & 520.00 \\
$23.0-23.9$ & 540.00 \\
$24.0-24.9$ & 560.00 \\
$25.0-25.9$ & 590.00 \\
$26.0-26.9$ & 610.00 \\
$27.0-27.9$ & 630.00 \\
\hline
\end{tabular}

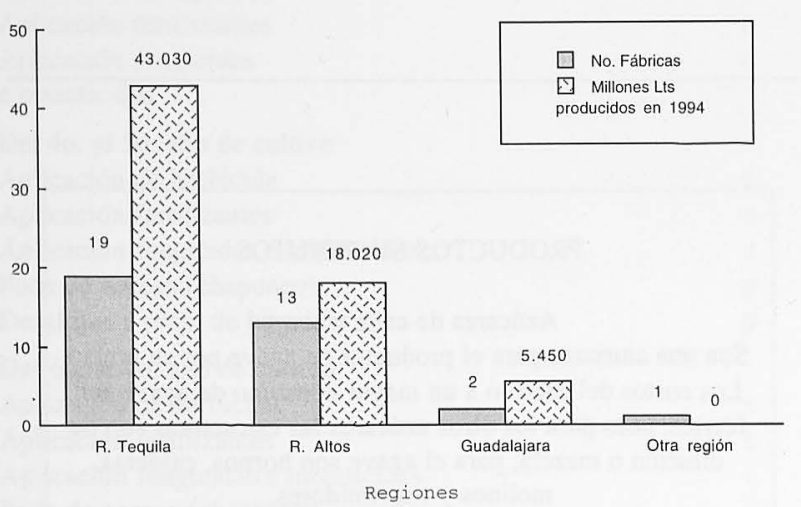

FIguRa 6. Distribución de las fábricas de tequila en Jalisco y su producción respectiva en millones de litros en 1994 (CRIT, 1994). quila blanco) o madurados (reposado y añejo). En la Figura 8 se observa el proceso industrial y los tipos de tequila que se producen según la Norma Oficial del tequila vigente (SECOFI, 1994).

Según la tendencia histórica de producción anual de tequila (Fig. 9) en el año 2000 ésta podría ser de 80 millones de litros (Fig. 10), es decir un 15\% más que la actual, y el mercado de exportación de 55 m.d.1., 21\% más del registrado en 1994. Si el mercado del tequila crece es de esperarse lo mismo del sector agrícola, pero no siempre es así, la adulteración de la bebida ha provocado la desconfianza y la inseguridad de que la demanda industrial de 220,000 toneladas anuales de agave (Siglo 21, 1995) se eleve, se mantenga o disminuya. La Secretaría de Agricultura, Ganadería y Desarrollo Rural, (Siglo 21, 1995) ha validado el inventario

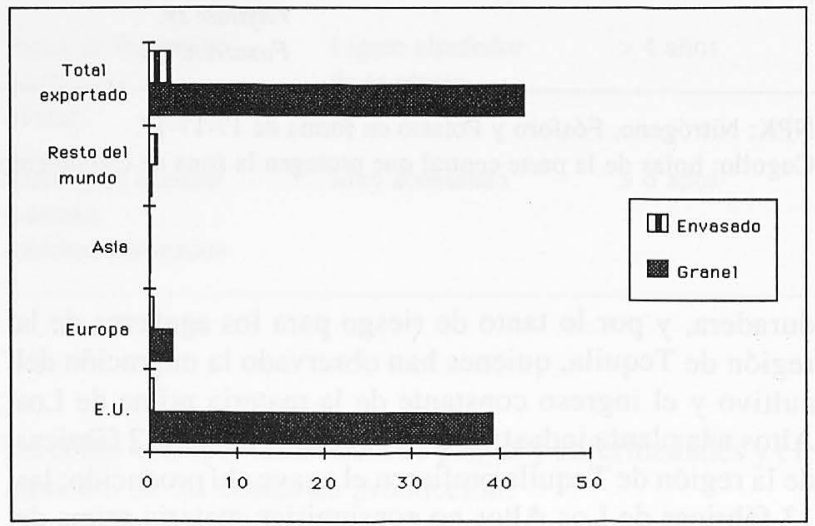

FIGURA 7. Zonas de exportación de tequila y formas de venta en 1994 (CRIT, 1994).

CUADRo 7. Cantidad de plantas en etapa de cosecha y sus equivalentes en toneladas (SAGDR y CRT, 1995) y precio del excedente de agave en el mercado.

\begin{tabular}{lllll}
\hline $\begin{array}{l}\text { Millones } \\
\text { de plantas }\end{array}$ & $\begin{array}{l}\text { Toneladas } \\
\text { sin mercado }\end{array}$ & $\begin{array}{l}\text { N\$ } \\
(350 / \text { ton })\end{array}$ & $\begin{array}{l}\text { Miles de plantas } \\
35 \mathrm{Kg} / \mathrm{pta})\end{array}$ & $\begin{array}{l}\text { Has. } \\
(300 / \mathrm{Ha})\end{array}$ \\
\hline 60.7 & 23,510 & $\begin{array}{l}8,228,500 \\
(\$ 1 \text { millón USA })\end{array}$ & 671,714 \\
\hline
\end{tabular}

Plantaciones establecidas de 1983 a 1987, con una edad de cultivo igual y mayor a 8 años. La información procede de los registros realizados por el Consejo Regulador del Tequila y la Secretaría de Agricultura, Ganadería y Desarrollo Rural en 1995.

CuAdro 8. Número de plantas y su edad de cultivo registradas ante el Consejo Regulador del Tequila (1995).

\begin{tabular}{lllll}
\hline $\begin{array}{l}\text { Edad del } \\
\text { cultivo }\end{array}$ & Etapa & $\begin{array}{l}\text { Plantaciones } \\
\text { actuales }\end{array}$ & $\begin{array}{l}\text { Millones } \\
\text { de plantas }\end{array}$ & Fenómeno \\
\hline 1-2 años & Inicial & $1994-93$ & 8.33 & $\begin{array}{l}\text { Desabasto } \\
\text { Equilibrio? }\end{array}$ \\
3-4 años & $\begin{array}{l}\text { Producción } \\
\text { de hijuelos }\end{array}$ & $1992-91$ & 34.80 & Sobreoferta? \\
5-7 años & A la Madurez & $1990-88$ & 68.80 & Sobreoferta \\
\hline 8 años & Cosecha & $1987-83$ & 60.70 & \\
\hline
\end{tabular}




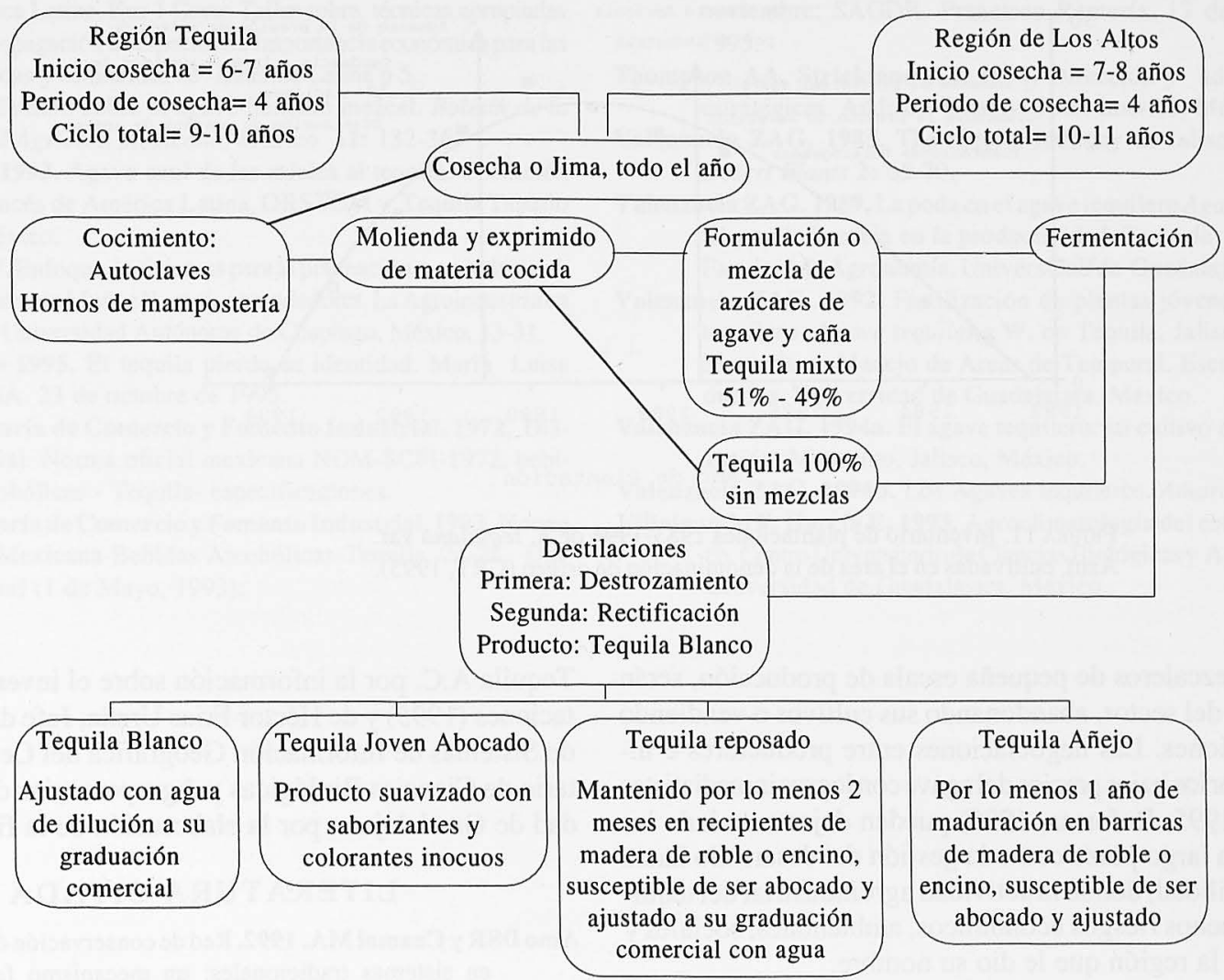

Figura 8. Proceso de producción de tequila.

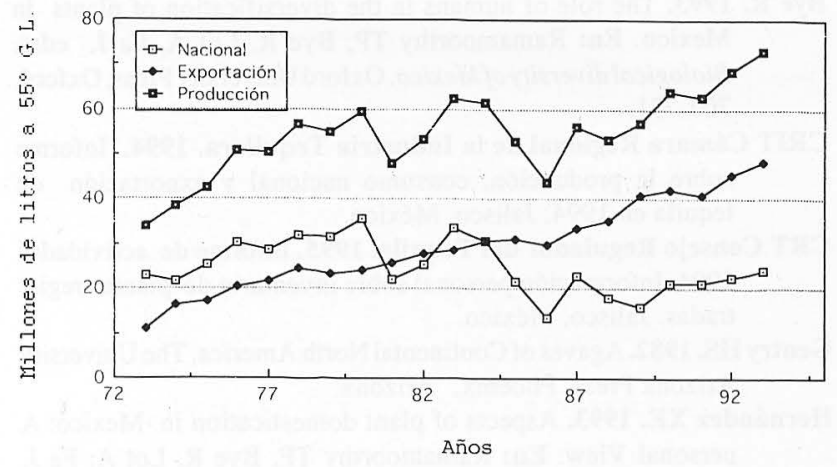

FIGURA 9. Producción de tequila y ventas del mercado nacional y de exportación de 1973 a 1993.

de agave del Consejo Regulador del Tequila (CRT, 1995) con una confiabilidad del $90 \%$ en sus datos (Fig. 11). La información oficial indica que hay un excedente de 23,510 ton (Cuadro 7), problemática que hoy se vive con una situación indefinida sobre las otras edades de plantación

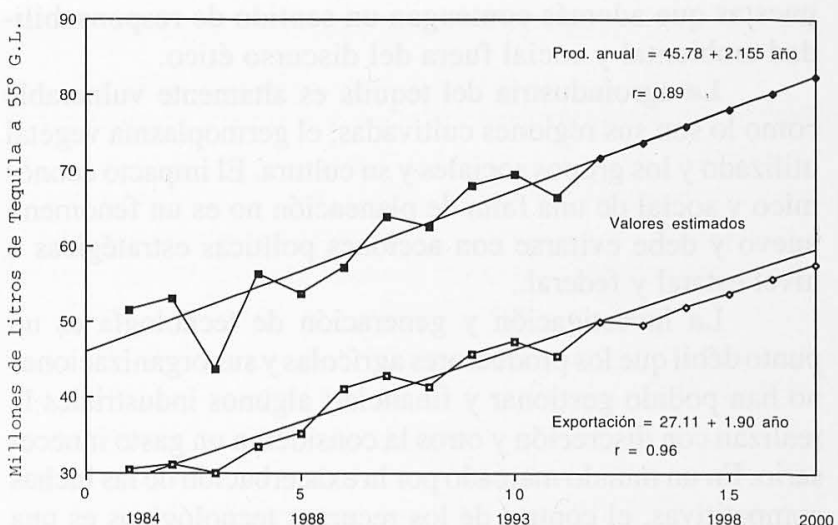

FIgURA 10. Tendencia histórica de la producción total y la exportación de tequila de 1984 a 1994 y la proyección estimada hasta el año 2000.

(Cuadro 8), donde el abastecimiento de la industria parece estar asegurado por encima de lo esperado.

Lo anteriormente expresado en el análisis de competitividad y en la demanda del producto, indican que los productores sin una relación directa con la integración industrial y los 


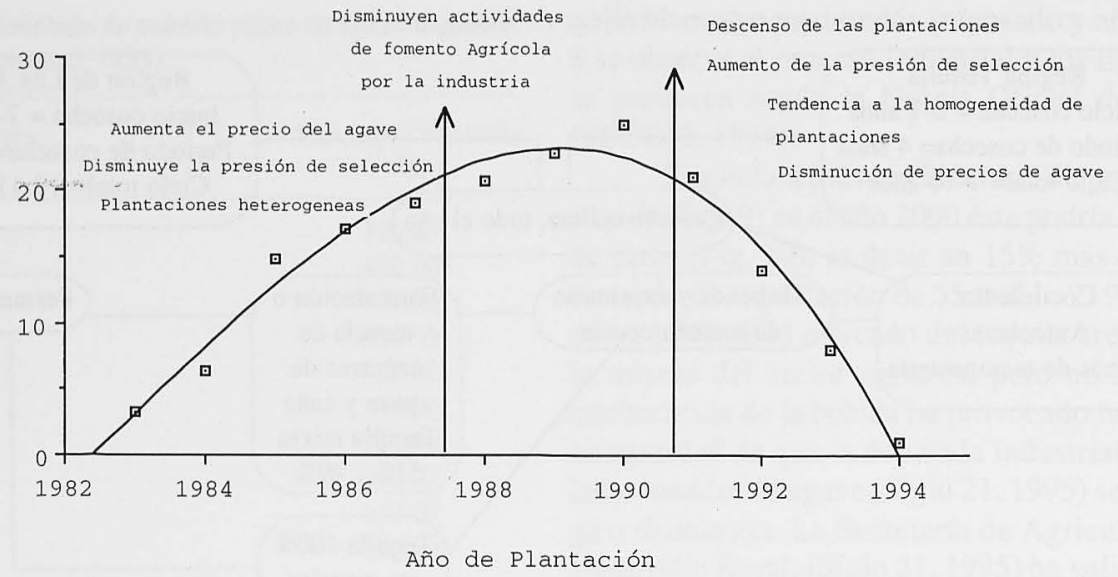

FIGURA 11. Inventario de plantaciones 1983-1994 de A. tequilana var. Azul, cultivadas en el área de la denominación de origen (CRT, 1995).

antiguos mezcaleros de pequeña escala de producción, serán expulsados del sector, abandonando sus cultivos o vendiendo sus plantaciones. Las negociaciones entre productores e industriales por los bajos precios del agave con logros inmediatistas (Siglo 21, 1995; Reforma, 1995) pueden dejar a un lado las estrategias a largo plazo como la gestión del desarrollo hacia la sustentabilidad; donde la actividad agroindustrial del tequila ofrezca menos riesgos económicos, ambientales, sociales y culturales a la región que le dio su nombre.

A manera de conclusiones puede advertirse un problema complejo que debe ser reconocido y tratado como tal, en donde un modelo lineal de pensamiento (causa-efecto) ofrece soluciones poco viables; desarticular el conocimiento de la problemática poco ayuda a plantear y construir propuestas que además contengan un sentido de responsabilidad ambiental y social fuera del discurso ético.

$\mathrm{La}$ agroindustria del tequila es altamente vulnerable como lo son sus regiones cultivadas, el germoplasma vegetal utilizado y los grupos sociales y su cultura. El impacto económico y social de una falta de planeación no es un fenómeno nuevo y debe evitarse con acciones políticas estratégicas a nivel estatal y federal.

La investigación y generación de tecnología es un punto débil que los productores agrícolas y sus organizaciones no han podido gestionar y financiar; algunos industriales la realizan con discreción y otros la consideran un gasto innecesario. En un mundo marcado por la exacerbación de las luchas competitivas, el control de los recursos tecnológicos es una capacidad fundamental estratégica (OCDE, 1995). La propuesta de atender una necesidad económica y social como la del agave tequilero, justifica la creación de un proyecto interinstitucional que estudie el recurso agave en toda su extensión, sus sistemas de producción y actividades económicas.

\section{AGRADECIMIENTOS}

Para este artículo se agradecen las importantes aportaciones de los Maestros: Mariín Martínez de Consejo Regulador del
Tequila A.C. por la información sobre el inventario de plantaciones (1995) y de Héctor Frias Ureña, Jefe del Laboratorio de Sistemas de Información Geográfica del Centro Universitario de Ciencias Biológicas y Agropecuarias de la Universidad de Guadalajara, por la elaboración de la figura 1.

\section{LITERATURA CITADA}

Amo DSR y Caamal MA. 1992. Red de conservación de germoplasma en sistemas tradicionales: un mecanismo factible para el mantenimiento de la biodiversidad. En: Silvia R. del Amo, coord. UNAM, SEDUE, SEP, México, 73-94.

Bustamante I. 1984. Estudio agroecológico de los agaves de la zona de influencia de Tequila, Jalisco. Facultad de Ciencias, ENEP, UNAM, México.

Bye R. 1993. The role of humans in the diversification of plants in Mexico. En: Ramamoorthy TP, Bye R, Lot A, Fa J, edrs. Biological diversity of Mexico. Oxford University Press, Oxford, 707-731.

CRIT Cámara Regional de la Industria Tequilera. 1994. Informe sobre la producción, consumo nacional y exportación de tequila en 1994. Jalisco, México.

C.RT Consejo Regulador del Tequila. 1995. Informe de actividades 1994. Información personal sobre inventario de plantas registradas. Jalisco, México.

Gentry HS. 1982. Agaves of Continental North America. The University Arizona Press, Phoenix, Arizona.

Hernández XE. 1993. Aspects of plant domestication in Mexico: A personal View. En: Ramamoorthy TP, Bye R, Lot A, Fa J, edrs. Biological diversity of Mexico. Oxford University Press, Oxford, 733-753.

Luna ZR. 1991. La historia del tequila, de sus regiones y sus hombres. Consejo Cultural para la Cultura y las Artes, México.

Muriá RJM. 1990. El tequila, boceto histórico de una industria. Cuadernos de Difusión Científica No. 18, Universidad de Guadalajara, México.

Nobel PS y Valenzuela VAG. 1987. Environmental responses and productivity of the CAM plant, Agave tequilana Agriculture Forest Meteorology 39: 319-334.

OCDE Organización para la Cooperación y Desarrollo Económico. 1995. Las pequeñas y medianas empresas. Tecnología y Competitividad. Ediciones Mundi-Prensa, México, 29-42. 
Palomino HG. 1994. Estado actual de especies de zonas áridas y semiáridas de América Latina. En: 1 Curso Taller sobre técnicas apropiadas para la propagación de especies de importancia económica para las zonas áridas y semiáridas de América Latina p 5.

Pérez L. 1887. Estudio sobre el agave llamado mezcal. Boletín de la Sociedad Agrícola Mexicana, México 11: 132-36.

Pla R, Tapia J. 1993. Agave azul de las mieles al tequila. Coedición Inst. Francés de América Latina, ORSTOM y Tequila Tapatio S.A., México.

Ramos OC. 1987. Enfoque de sistemas para la planeación agroindustrial. En: Manrrubio Muñoz R. et al., compiladores. La Agroindustria en México. Universidad Autónoma de Chapingo, México, 13-31.

Reforma Diario 1995. El tequila pierde su identidad. Maria Luisa Alós. 36A. 23 de octubre de 1995.

SECOFI Secretaría de Comercio y Fomento Industrial. 1972. Diario Oficial. Norma oficial mexicana NOM-SCFI-1972, bebidas alcohólicas - Tequila- especificaciones.

SECOFI Secretaría de Comercio y Fomento Industrial. 1993. Norma Oficial Mexicana-Bebidas Alcohólicas-Tequila. 24-28. Diario Oficial (1 de Mayo, 1993).
Siglo 21 Diario, 1995. La sobreproducción de agave terminará en noviembre: SAGDR. Francisco Rentería. 17 de octubre de 1995.

Thompson AA, Strickland AJ. 1994. Dirección y administración estratégicas. Addison-Wesley- Iberoamérica, México.

Valenzuela ZAG. 1985. The tequila Industry in Jalisco, México. Desert Plants 2: 65-70.

Valenzuela ZAG. 1987. La poda en el agave tequilero Agave tequilana W.y su influencia en la productividad. Tesis de licenciatura. Facultad de Agronomía, Universidad de Guadalajara, México.

Valenzuela ZAG. 1992. Fertilización de plantas jóvenes de agave tequilero Agave tequilana W. en Tequila, Jalisco. Tesis de Maestría en Manejo de Areas de Temporal. Escuela de Graduados. Universidad de Guadalajara, México.

Valenzuela ZAG. 1994a. El agave tequilero: su cultivo e industrialización. Monsanto, Jalisco, México.

Valenzuela ZAG. 1994b. Los Agaves tequileros. Amaranto 7: 1-8.

Villalpando F, García E. 1993. Agroclimatología del estado de Jalisco, Centro Universitario de Ciencias Biológicas y Agropecuarias. Universidad de Guadalajara, México. 